This is an open access article under the terms of the Creative Commons Attribution-NonCommercial License, which permits use, distribution and reproduction in any medium, provided the original work is properly cited and is not used for commercial purposes.

\title{
Reorganization of taxonomic, functional, and phylogenetic ant biodiversity after conversion to rubber plantation
}

\author{
Cong Liu, ${ }^{1,5}$ Benoit Guénard, ${ }^{1,2}$ Benjamin Blanchard, ${ }^{1,3}$ Yan-Qiong Peng, ${ }^{4}$ and Evan P. Economo ${ }^{1}$ \\ ${ }^{1}$ Okinawa Institute of Science and Technology Graduate University, Okinawa, 904-0495, Japan \\ ${ }^{2}$ School of Biological Sciences, The University of Hong Kong, Pokfulam Road, Hong Kong, China \\ ${ }^{3}$ Committee on Evolutionary Biology, University of Chicago, Chicago, Illinois, 60637 USA \\ ${ }^{4}$ Key Laboratory of Tropical Forest Ecology, Xishuangbanna Tropical Botanical Garden, Chinese Academy of Sciences, \\ Kunming, China
}

\begin{abstract}
Agricultural activity associated with habitat conversion is a major driver of biodiversity loss across the tropics. The decline of species richness is a common outcome of conversion to agricultural land use, but the associated changes in functional and phylogenetic diversities, and spatial patterns of beta diversity, are not well understood. These patterns may shed light on underlying ecological processes that are of both basic and applied interest; for example the relative roles of stochastic assembly, ecological filtering, and competition in structuring ecological communities as well as broader consequences for ecological functioning in agroecosystems. Here we investigate the effects of conversion to rubber plantation (Hevea brasiliensis), a rapidly spreading agroecosystem in Southeast Asia, on leaf-litter ant taxonomic, functional, and phylogenetic diversities at local and among-sites scales in Xishuangbanna, Southeast China. We found a sharp decline of ant species richness in rubber plantations compared with nearby forest habitat, with low beta diversity indicating spatial homogeneity of communities in rubber plantations. In addition, patterns of both functional alpha and beta diversities suggested the emergence of a functionally distinct ant community in the agroecosystem compared to the forest habitats. These results support a role of ecological filtering in structuring the taxonomic and functional composition of both rubber and forest habitats. In contrast, changes in phylogenetic diversity were modest and not significantly different from random expectations despite strong phylogenetic signal of functional traits. This study highlights the need for a pluralistic approach to characterizing the loss of biodiversity in agroecosystems, as well as understanding the underlying mechanisms of community assembly driving this biodiversity loss.
\end{abstract}

Key words: biodiversity; community assembly; environmental filtering; Formicidae; functional diversity; Hevea brasiliensis; phylogenetic diversity; rubber plantation; Xishuangbanna.

\section{INTRODUCTION}

The current era of human domination of Earth's ecosystems has led to anthropocentric conversion of a significant proportion of Earth's surface to agroecosystems. The expansion of agricultural activities is driving an increasing loss of tropical forest (Hansen et al. 2013) and is likely to have serious impacts on biodiversity worldwide (Laurance et al. 2014). In Southeast Asia, agroecosystems such as oil palm plantations cover a large area and continue to expand, resulting in dramatic impacts on biodiversity (e.g., Fayle et al. 2010). In recent years the "Rubber Juggernaut" has been expanding rapidly, largely by replacing natural forest in Southeast Asia (Ziegler et al. 2009, Warren-Thomas et al. 2015). The implications of the emergence of this new habitat for both ecological processes and biodiversity conservation are not yet well understood. Here, we investigate the organization

Manuscript received 8 August 2015; revised 20 November 2015; accepted 6 January 2016. Corresponding Editor: I. Perfecto.

${ }^{5}$ E-mail: cong.liu0514@gmail.com of ant biodiversity, an ecologically dominant component of these ecosystems, in a mixed landscape of forest and rubber habitat in Southeast Asia.

For many taxa, a decline of species richness is a common outcome of conversion of forests to agroecosystems (Philpott et al. 2008a), including rubber plantations (Meng et al. 2012). However, the responses of other dimensions of biodiversity, such as functional and phylogenetic diversity, and changes in the organization of biodiversity in space (e.g., beta diversity), are not well documented or understood. The nature of these responses has important implications for our understanding of the community ecology of both natural and agricultural ecosystems (Cavender-Bares et al. 2009, Swenson et al. 2012, Weinstein et al. 2014).

If species richness declines with conversion from forests to rubber plantation, a natural question is whether the new species assemblages are random with respect to species identity, functional traits, and phylogeny. This question sheds light on a broader debate in ecology, the extent to which ecological communities are structured 
by stochastic factors such as ecological drift (Hubbell 2001), or more deterministic assembly forces (Chase and Leibold 2003). In the former view, species differences (including functional and phylogenetic differences) are not important in determining which species occupy which communities. Although rubber habitats may have reduced functional and phylogenetic diversity due to reduced richness, these reductions should be consistent with random expectations. In this scenario, beta diversity should also mainly follow random expectations, where overlap between spatially separated rubber habitats is consistent with null models.

Under a deterministic scenario, various nonrandom processes, such as competition and species sorting, could structure communities (Chase and Liebold 2003). For example, if rubber habitat is acting as an ecological filter, then only a subset of forest species may colonize and survive there. This leads to a decline of beta diversity (within habitat type) along with the decline in alpha diversity, because the same subset of forest species should be found in rubber habitat in even spatially disparate locations. This diversity decline may be associated with a clustered functional structure with reduced functional alpha and beta diversity if the habitat is filtering a subset of functional traits (Kraft et al. 2007). Phylogenetic diversity may also be reduced if the species able to persist in rubber plantations are clustered on the phylogeny. This could occur either if functional traits have some phylogenetic signal or because the ecological filter effect is acting on other non-functional traits with phylogenetic signal (Srivastava et al. 2012).

Another deterministic hypothesis involves a strong role of competition in structuring communities (Kunstler et al. 2012). If competition limits the coexistence of similar species, then the remaining communities should be overdispersed in functional and/or phylogenetic space (Lovette and Hochachka 2006). Thus, while species richness may be reduced in rubber plantation, those species are more functionally or phylogenetically disparate than random expectations. It is worth noting that mutualisms, host-pathogen interactions, plant-insect interactions, or other density-dependent processes have also been hypothesized to lead to patterns of clustering and/or overdispersion deviating from null expectations (Cavender-Bares et al. 2009).

Whatever the mechanisms driving community assembly, changes in ant community functional structure in agroecosystems have potentially important consequences. Functional diversity has been defined as "the value and range of those species and organismal traits that influence ecosystem functioning" (Tilman 2001). It links species diversity and ecosystem functioning, and thus could be a tool to predict the functional consequences of agricultural activities (Petchey and Gaston 2006). For example, researchers have found that high agricultural intensification could reduce functional diversity of mammals and birds at a higher rate than the loss of species richness, indicating a disproportionate effect on ecosystem func- tioning (Flynn et al. 2009). The low level of ant functional redundancy in tropical forest indicates that the species loss in secondary forest may cause more severe declines of ecosystem functioning (Bihn et al. 2010).

More generally, ants have been used as environmental indicator in previous studies where they are a powerful monitoring tool for studying the effects of humaninduced habitat change thanks to their abundance, ease of sampling, and sensitivity to environmental disturbance (Andersen and Majer 2004). Moreover, ants play an important role in ecosystem functioning (Bihn et al. 2010, Ewers et al. 2015), such as biological control (Philpott et al. 2008b), seed dispersal (Levey and Byrne 1993), ecosystem engineering (Hölldobler and Wilson 1990), and nutrient cycling (Del Toro et al. 2012).

Our study area near Xishuangbanna, Yunnan, China, is located within the Indo-Burma biodiversity hotspot (Myers et al. 2000), and harbors a large fraction of Chinese biodiversity in its tropical landscape (Yang et al. 2004, Liu et al. 2015). Like many parts of Southeast Asia, the expansion of rubber plantation is dramatically changing the landscape in this area (Liu and Slik 2014), making it a pressing need to understand implications for biodiversity. Ant community composition in rubber plantations has not been well characterized in the region, with (to our knowledge) only one thorough ant survey performed to date (Hosoishi et al. 2013). That study, from an agriculture-dominated landscape in Cambodia, found a much simplified ant community in rubber habitat with exotic species taking a prominent role. The Xingshuabanna area provides an interesting contrast as it is composed of a mosaic landscape with natural forest interspersed with dominant rubber plantations and other minor crops (e.g., banana plantations).

We analyzed the effects of conversion of forest to rubber plantation on the alpha, beta, and gamma diversities of ground ant communities, with three overall aims. First, we sought to characterize changes across the taxonomic, functional, and phylogenetic dimensions of biodiversity. Second, we tested the hypothesis that conversion to rubber plantation facilitated the establishment and dominance of exotic species, as has been observed in a previous study (Hosoishi et al. 2013). Third, we tested three hypotheses describing community processes underlying changes in biodiversity in the agroecosystem: (1) Stochastic assembly: if the ant community is structured by stochastic processes in rubber plantations, we expected that the loss of functional and phylogenetic diversities are consistent with null expectations at both local (alpha diversity) and landscape scale (gamma diversity). The taxonomic, functional, and phylogenetic beta diversities will also be consistent with null expectation. (2) Environmental filtering: If environmental filtering plays an important role of structuring the ant community in rubber plantations, we expect that ant communities would be functional and phylogenetically clustered, as would be expected if traits are phylogenetically conserved at both local (alpha diversity) and 
landscape (gamma diversity) scales. Moreover, ant assemblies in the similar environment such as rubber plantation will lead to low taxonomic, functional, phylogenetic beta diversity. (3) Biotic structuring: If biotic processes such as competition primarily shape ant community structure, communities in rubber plantations would be functionally and phylogenetically overdispersed in local (alpha) and landscape (gamma) scales.

\section{Methods \\ Study site}

This study was carried out in Xishuangbanna prefecture $\left(21^{\circ} 55^{\prime} \mathrm{N}, 101^{\circ} 15^{\prime} \mathrm{E}\right)$ located in Yunnan Province, a tropical region in southern China. In this area, rubber plantations have expanded dramatically during the past 20 years due to the massive expansion of smallholder rubber farms, and have become the main driver of local habitat loss and fragmentation (Liu and Slik 2014).

\section{Sampling of ants}

In July 2013, we sampled leaf litter ants from 11 sites in rubber plantation habitat and 24 sites from forest habitats. The distance between sites varies between $200 \mathrm{~m}$ and $10 \mathrm{~km}$. At each sampling site, we established a $20 \mathrm{~m}$ per side quadrat $\left(400 \mathrm{~m}^{2}\right)$ and collected leaf litter samples (from $1-\mathrm{m}^{2}$ quadrats) at the four corners of the $400-\mathrm{m}^{2}$ quadrat. Furthermore, leaf litter within the $400-\mathrm{m}^{2}$ quadrat was also collected at 12 sub-sites to cover the variety of available microhabitats available (e.g., base of a large tree, near decaying wood, under on accumulation of leaf litter, near more open leaf litter habitats, etc.) and obtain more accurate data on species composition and richness of each site. Ants were collected and extracted by mini Winkler extractors for $72 \mathrm{~h}$ using the shuffling method as described in Guénard and Lucky (2011) to limit sampling artifacts.

Ant samples were sorted to morphospecies, pointmounted, and identified to species or, if undescribed, assigned a standardized morphospecies code. Details on the species detected and their identifications, as well as their habitats are provided (Appendix S1). All mounted and alcohol-preserved ant specimens are currently located in E. P. Economo's collection at the Okinawa Institute of Science and Technology Graduate University.

\section{Functional traits}

Our aim was to quantify ant functional diversity through morphological traits relevant to resource use and microhabitat preference. Although using traits to characterize the functional roles of species undoubtedly misses some aspects of species' ecologies, direct links have been demonstrated between functional traits and diet, foraging pattern, and habitat preference (Gibb et al. 2015). The functional traits measured for each species were as follows.
Weber's length.-Measured as the maximum length from the anterior edge of the pronotum to the posterior edge of the propodeum. We measured Weber's length as the indicator of total body size, which related to many ecological life history traits such as resource use (Weiser and Kaspari 2006).

Relative eye length.-Eye size is related to ant food searching and navigation (Weiser and Kaspari 2006). Eye size could also be an indication of habitat occupation since ants living underground likely do not need vision as much as ants living above the ground do (Keller 2011). Relative eye length was measured as the ratio of eye length to mesosoma length.

Relative scape length.-Scape length might have important effects on receiving chemosensory information (Weiser and Kaspari 2006). Ants with long scape length may be more sensitive to pheromone trails (Weiser and Kaspari 2006). We measured relative scape length as the ratio of scape length to mesosoma length.

Relative mandible length. - Ant mandible length might indicate specialization in a predatory role, and thus can have important effects on the type of resources consumed (Weiser and Kaspari 2006). Relative mandible length was measured as the ratio of mandible length (the maximum length from the basal margin to the apical tooth of the mandible) to mesosoma length.

Relative leg length. - Longer legs allow faster and more efficient locomotion and foraging (Weiser and Kaspari 2006). Relative leg length was measured as the ratio of leg length (hind femur + hind tibia) to mesosoma length.

For all ant trait measurements, up to five randomly selected individuals of each species were measured. Only minor workers were measured if the ant species has distinct major and minor workers. In total, 754 workers representing 186 leaf litter ant species were measured.

\section{Community phylogeny}

To construct a community phylogeny, we used posterior sets of ant-wide phylogenies generated during in a separate project (Economo et al., unpublished manuscript). These trees share many of the features of other ant wide phylogenies (Moreau et al. 2006, Moreau and Bell 2013) but incorporate the latest molecular data generated during subfamily-level phylogenetic work (Brady et al. 2014, Ward et al. 2015) to maximize coverage of genera. The tree sets capture relationships between subfamilies and genera, but structure within trees follow a random branching process to resolve polytomies, similar to other efforts using mammals (Kuhn et al. 2011), producing a set of trees that collectively integrate over phylogenetic uncertainty. The trees were initially constructed to match richness of species in each genus, but then pruned to only include the number of 
species in each genus we detected in our study. We used 100 of these trees taken from the posterior sets, and over all these trees the phylogenetic distance between two congeneric species reflects the average divergence between species of that genus although the position of species varies within genera among individual trees. While the phylogenetic structure at the genus level and above captures the majority of phylogenetic structure of these communities, our results are limited to signal at these deeper phylogenetic scales due to the lack of resolution of recent phylogenetic structure. For the analyses in this paper, both described and undescribed species were placed randomly into genera, and before analysis the tree was pruned to only include taxa collected in the field surveys. All phylogenetic analyses such as phylogenetic signal, phylogenetic alpha, beta, and gamma diversity calculations were performed on all 100 trees.

\section{Phylogenetic signal}

Blomberg's $K$ (Blomberg et al. 2003) and Pagel's $\lambda$ (Pagel 1999) were used to investigate if there is phylogenetic signal for habitat preference (forest vs. rubber plantation), as well as the five morphological traits. Both indices test whether an observed distribution of traits differs from expected with the traits evolving under Brownian model (Pagel 1999, Blomberg et al. 2003, Münkemüller et al. 2012). For both Blomberg's $K$ and Pagel's $\lambda$, a value of 1 indicates phylogenetic signal with traits evolved under Brownian model, whereas a value close to 0 indicates no phylogenetic signal. The range of Pagel's $\lambda$ is from 0 to 1 , however, the value of Blomberg's $K$ can be greater than 1 , indicating strong phylogenetic signal and traits conservatism.

We calculated $K$ and $\lambda$ values for each traits and habitats for the 100-tree set. To evaluate the statistical significance of the presence phylogenetic signal, we first compared the observed $K$ values to the $K$ values of null distribution generated by simulating random traits on the phylogeny; second, we estimated the probability of the observed $\lambda$ differs the null hypothesis of $\lambda$ equal to 0 (no phylogenetic signal) using likelihood ratio test. Phylogenetic signals (Blomberg's $K$ and Pagel's $\lambda$ ), and their statistical significance were calculated using phylosig function in R package phytools (Revell 2012).

\section{Estimation of taxonomic diversity}

Taxonomic alpha and gamma diversity.-We used species richness (the number of ant species in each site) as an indicator of taxonomic diversity. Taxonomic gamma diversities in forest and rubber plantation were simply represented by the total number of ant species collected in each habitat. As ants are super organisms and often encountered/sampled in whole colonies, diversity metrics based on individual abundances can be problematic (Gotelli et al. 2011).
Taxonomic beta diversity.-The Raup-Crick dissimilarity metric $\left(\beta_{\mathrm{rc}}\right)$ was used to calculate taxonomic beta diversity of ant communities within forest and rubber plantations. $\beta_{\mathrm{rc}}$ is a null-model-based metric that is alpha diversity independent (Chase et al. 2011). $\beta_{\text {rc }}$ ranges from -1 to 1 , indicating that the observed dissimilarity of two communities is lower than (reaching -1), higher than (reaching 1), or similar (reaching 0 ) to what is expected by chance. Thus, the mean value of $\beta_{\text {rc }}$ among all sites in one habitat that is close to 0 indicates a stochastic assembly, while a mean value higher or lower than 0 suggests deterministic community assembly (see also Püttker et al. [2015] for further details).

We compared the mean $\beta_{\mathrm{rc}}$ based on ant presenceabsence data among sites within forest and rubber plantation habitats to investigate the main driver of species coexistence in rubber plantations. Since the delineation of the species pool is very important for calculating the Raup-Crick metric (Chase et al. 2011), we identified the species pool as all ant species we collected in this study since they are potentially able to colonize the sampling sites. We calculated $\beta_{\mathrm{rc}}$ using the $\mathrm{R}$ script provided by Chase et al. (2011). To visualize taxonomic beta diversity, we performed a two-dimensional nonparametric multidimensional scaling (NMDS) based on the value of $\beta_{\mathrm{rc}}$ between sites using $\mathrm{R}$ package Vegan (Oksanen et al. 2015).

In order to test whether the average $\beta_{\mathrm{rc}}$ is significantly different from random expectation, we compared the observed $\beta_{\text {rc }}$ in forest, rubber, and rubber vs. forest (rubber $\times$ forest) to the $\beta_{\text {rc }}$ of 1000 randomized ant communities of the same species richness for each sites using Wilcoxon Mann-Whitney test in R 3.0.2 (R Development Core Team 2015). Randomized communities were generated by randomizing the community data matrix using the "Independent Swap" approach, following the R code described in Swenson (2014).

\section{Estimation of functional and phylogenetic diversity}

The following analyses of functional and phylogenetic alpha, beta, and gamma diversity (FD, PD, MPD, MNTD, $D_{\mathrm{pw}}$, and $D_{\mathrm{nn}}$ ) were calculated following Swenson (2014) by using R package Picante (Kembel et al. 2010).

Functional and phylogenetic alpha diversity.-Functional alpha diversity of ant communities in each sampling site was calculated using Petchey and Gaston's FD (Petchey and Gaston 2006). In order to calculate FD, the $z$-standardized trait values were converted into a Euclidean distance matrix, and then were clustered to produce a trait dendrogram representing the functional relationship among ant species using UPGMA method (see Mouchet et al. 2008 for further details on the distance and clustering algorithms). We chose the combination of Euclidean distance and UPGMA clustering because they gave the highest cophenetic cor- 
relation coefficient between the original distances and the distances estimated form the resulting dendrogram (0.87). The Euclidean distance matrix generation and UPGMA clustering were conducted by $\mathrm{R}$ functions dist and hclust, respectively. For each site, we summed the branch lengths of the dendrogram corresponding to species present to calculate FD.

Phylogenetic alpha diversity was calculated using Faith's phylogenetic diversity (PD; Faith 1992), mean pairwise distance and mean nearest taxon distance (MPD and MNTD; Webb et al. 2002).

Standardized effect sizes (SES) of functional alpha diversity $\left(\mathrm{SES}_{\mathrm{FD}}\right)$ and phylogenetic alpha diversity $\left(\mathrm{SES}_{\mathrm{PD}}, \mathrm{SES}_{\mathrm{MPD}}\right.$, and $\mathrm{SES}_{\mathrm{MNTD}}$ ) were calculated using the SES formula as follows in order to detect the differences between observed values vs. communities generated by null models:

$$
\mathrm{SES}=\left(\text { Mean }_{\mathrm{obs}}-\mathrm{Mean}_{\text {null }}\right) / \text { s.d. }_{\text {null }} .
$$

The standardized effect sizes of MPD (SES ${ }_{\mathrm{MPD}}$ ) and MNTD $\left(\mathrm{SES}_{\mathrm{MNTD}}\right)$ are equivalent to net relatedness index (NRI), and the nearest taxon index (NTI). We multiplied NRI and NTI by -1 , and therefore, negative value indicates phylogenetic clustering, and positive value represents phylogenetic overdispersion.

Null model communities for analyzing functional and phylogenetic alpha diversities were generated by randomizing the community data matrix using "Independent Swap" approach for 1000 times following the R code in Swenson (2014).

Functional and phylogenetic beta diversity.-Both functional and phylogenetic beta diversity was calculated using two distance-based measurement metrics, the present-absent weight pairwise distance metric $\left(D_{\mathrm{pw}}\right)$, and the present-absent weight nearest neighbor distance metric $\left(D_{\mathrm{nn}}\right.$; Swenson 2011a). $D_{\mathrm{pw}}$ generally reflect the overall dissimilarity between communities, while $D_{\mathrm{nn}}$ is likely better for qualifying the patterns among close related species between different communities (Swenson 2011a). Standardized effect sizes of functional and phylogenetic beta diversities (functional $\mathrm{SES}_{D \mathrm{pw}}$ and $\mathrm{SES}_{D \mathrm{nn}}$; phylogenetic $\mathrm{SES}_{D \mathrm{pw}}$ and $\mathrm{SES}_{D \mathrm{nn}}$ ) were also calculated using SES formula mentioned before.

Null model communities for analyzing functional and phylogenetic beta diversities were generated by randomly shuffling the names of taxa cross the traits matrix or the tips of phylogenetic tree for 1000 times following the $\mathrm{R}$ code in Swenson (2014). Two-dimensional nonparametric multidimensional scaling (NMDS) was also used to visualize the standardized effect sizes of functional and phylogenetic beta diversities.

Functional and phylogenetic gamma diversity.-Functional gamma diversity was calculated as the Petchey and Gaston's FD of all the species in each habitat. For phylogenetic gamma diversity, we calculated the Faith's PD of all the species collected in each habitat for 100 trees. We then compared the observed functional and phylogenetic gamma diversities in both habitats to the functional and phylogenetic gamma diversities of 1000 randomized ant communities of the same species richness (forest, $n=163$ species; rubber plantation, $n=83$ species).

Statistical significance tests.-A Wilcoxon MannWhitney test was used to investigate the statistical significance of the average functional and phylogenetic alpha diversity compare to null model. We used Welch's two-sample $t$ test to investigate whether the observed functional and phylogenetic beta diversity, as well as phylogenetic gamma diversity in each habitat is significantly different from the null expectation. All calculations and analyses were conducted in R 3.0.2.

\section{RESULTS}

We collected 186 ant species from 52 genera with our field sampling. Among them, 163 species from 48 genera were found in forest and 83 species from 38 genera were found in rubber plantations. According to the classification provided in Guénard and Dunn (2012), six potentially exotic species were found in our collection. Among them, Anoplolepis gracilipes Smith, Strumigenys membranifera (Emery), and Tetramorium tonganum Mayr were only found in one site of a rubber plantation. Tapinoma melanocephalum Fabricius, Technomyrmex albipes Smith, and Monomorium pharaonis Linnaeus were found in both natural forest and rubber plantations, however, their occurrences were very low compared to other native ant species (Appendix S1). All the results presented below are based on using all captured 186 species as a regional species pool.

\section{Taxonomic diversity}

Leaf litter ant species richness declined dramatically in rubber plantation with an average of 32 species (31.7 \pm 0.98 [mean \pm s.d.]) found in forest habitat sites and only $23(23.1 \pm 0.87)$ species found in rubber plantation habitat (Fig. 1A).

Mean beta diversity $\left(\beta_{\mathrm{rc}}\right)$ between sites in rubber plantations was lower than in forest habitats $(-0.78$ vs. -0.31 ), and $\beta_{\mathrm{rc}}$ across the two habitats was higher than within each habitat (Fig. 2A). Despite the variation observed between pairs of sites, the mean $\beta_{\mathrm{rc}}$ between sites in rubber plantation was more similar than expected by chance $(P<0.001$, Mann-Whitney test; Fig. 2A). However, the mean $\beta_{\mathrm{rc}}$ in forest and the mean $\beta_{\mathrm{rc}}$ across the two habitats were not significantly different from null expectation $(P=0.94$ and $P=0.16$ respectively, Mann-Whitney test; Fig. 2A). There is a clear separation between forest and rubber plantation in the NMDS plot (Fig. 2D). These results indicate that ant communities in rubber plantations were composed of a nonrandom subset of forest species and, as a result, rubber plantation 

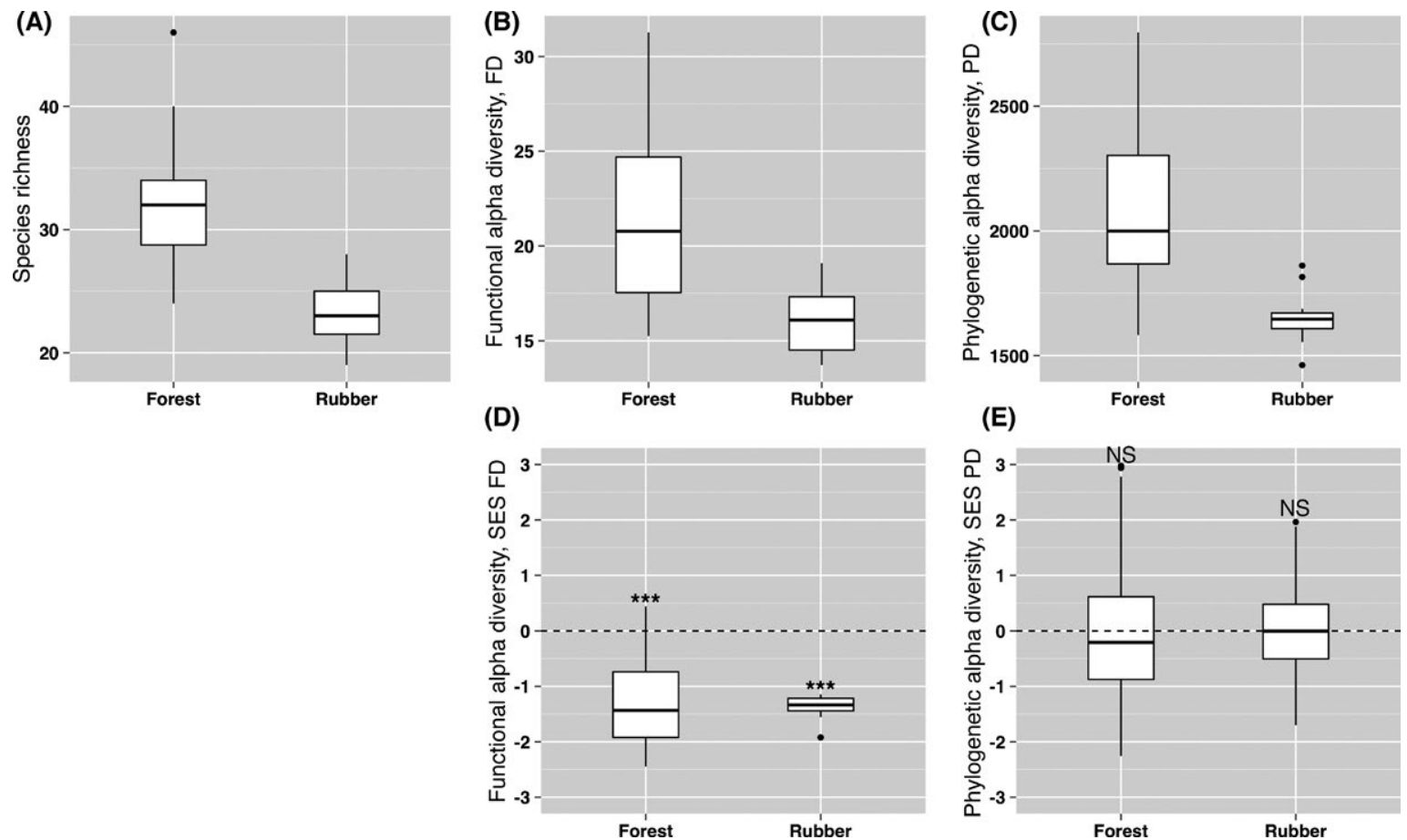

FIG. 1. Ant alpha diversities in forest and rubber plantation. (A) Ant species richness, (B) observed functional diversity (Petchey and Gaston's FD), and (C) observed phylogenetic diversity (Faith's PD) declined in rubber plantation. After correcting for species richness, functional diversity declined more than a null expectation based on randomly assembled communities in both habitats (D, scaled by SES FD), while phylogenetic diversity in both habitats was consistent with null expectation (E, scaled by SES PD). Asterisks indicate statistical significance $(* * * P<0.001$, NS, not significant; Mann-Whitney test between observed distribution and null distribution of FD and PD). The box encloses the 25-75th percentiles of the values, the whiskers extend to 1.5 times the interquartile range.

ant communities were more similar to each other than expected by chance.

\section{Functional diversity}

With the decline in species richness, there was a sharp decline of functional diversity as well (Fig. 1B). Moreover, FD declined more than the null expectation based on randomly assembled communities with the same reduced species richness $(P<0.001$ in both habitats, Wilcoxon Mann-Whitney test; Fig. 1D). In the aggregate, the species in rubber plantations showed significantly lower functional gamma diversity compared to randomly generated communities, however, ant species in forests exhibited a functional gamma diversity comparable to the null model (Fig. 3B and C). Those results indicate that the ant functional traits are clustered at a local and landscape scales in rubber plantations, with functional unique species being lost relative to forest habitats.

Functional beta diversity (the pairwise metric, functional $D_{\mathrm{pw}}$ ) was lower than the null expectation in both rubber and forest habitat (with both $P<0.001$, Welch's two-sample $t$ test; Fig. 2B), while beta diversity across the two habitats was higher than within each habitat and more consistent with null expectations $(P=0.732$,
Welch's two-sample $t$ test). A clear separation between ant community in forest and rubber plantation in the NMDS plot was also found (Fig. 2E). We found similar pattern of functional beta diversity by using the nearest-neighbor metric (functional $D_{\mathrm{nn}}$, Appendix S2: Fig. S1).

\section{Phylogenetic diversity}

To the extent that functional traits reflect evolutionary history, phylogenetic patterns may reflect patterns of functional diversity. We made a "co-phylogeny" plot between phylogeny and the functional dendrogram of 184 ant species in this study to show the relationship between phylogenetic and functional patterns (Fig. 4).

We measured the phylogenetic signal for habitat preference and the five functional traits we used by using of Blomberg's $K$ and Pagel's $\lambda$. In general, results of both indices were consistent with each other, except only one case (forest; Fig. 5). All the functional traits exhibited a phylogenetic signal using either Blomberg' $K$ or Pagel's $\lambda$ (Fig. 5), indicating that the functional traits used in this study were conserved phylogenetically. For habitat preference, ant communities in forest habitats exhibited a significant nonrandom phylogenetic pattern when calculated with Pagel's $\lambda$, but not with Blomberg's $K$ (Fig. 5); 

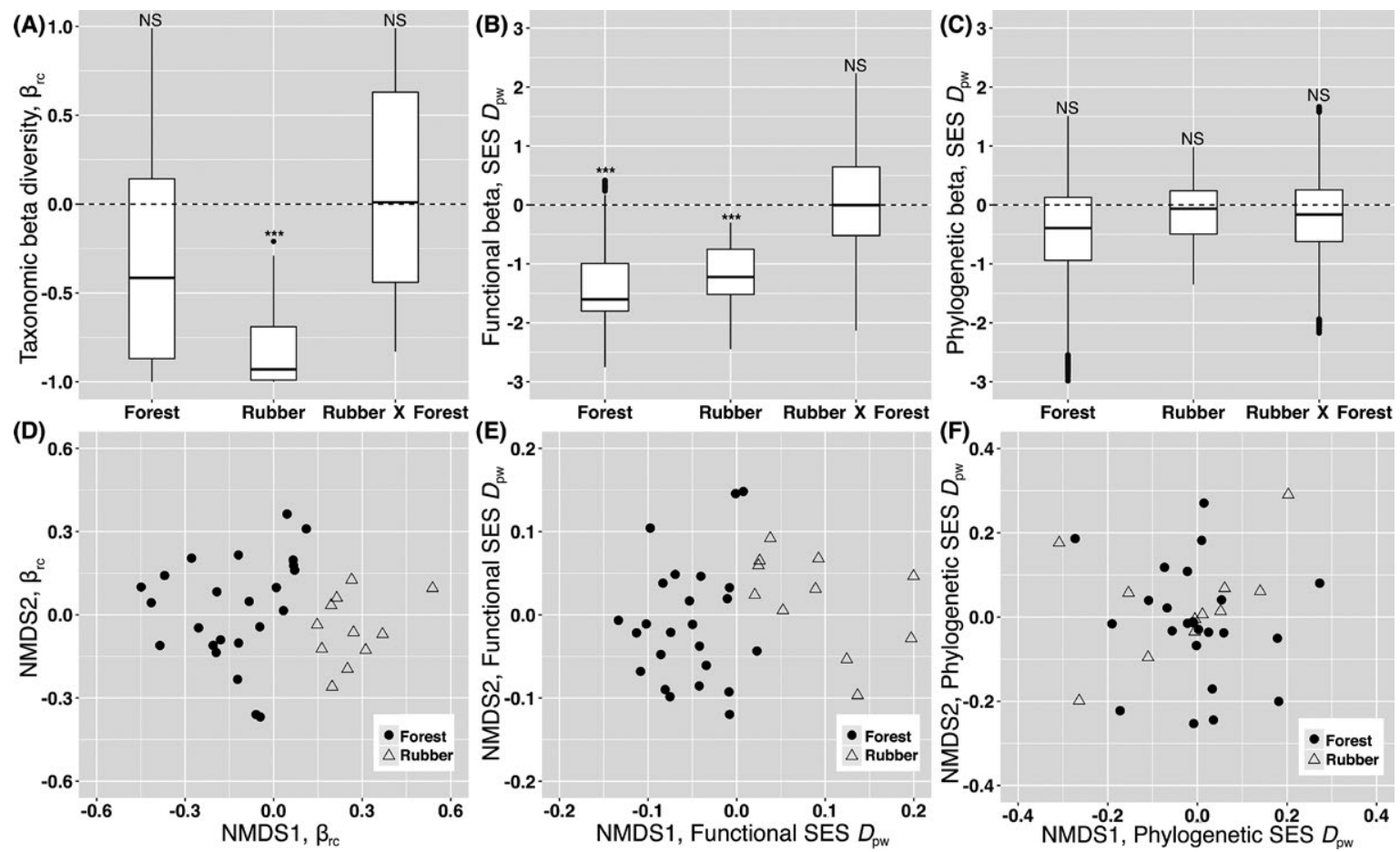

FIG. 2. Ant beta diversities between pairs of local communities in forest and rubber plantation and beta diversities between ant communities across forest and rubber plantation (rubber $\times$ forest). (A) Taxonomic beta diversity $\left(\beta_{\mathrm{rc}}\right)$; (B) functional beta diversity (represented by SES $D_{\mathrm{pw}}$ ); (C) phylogenetic beta diversity (represented by SES $D_{\mathrm{pw}}$ ). (D, E, F) Nonmetric multidimensional scaling (NMDS) ordination of sites within forest and rubber plantation based on different beta diversity indices ( $\beta_{\mathrm{rc}}$, functional SES $D_{\mathrm{pw}}$, and phylogenetic SES $D_{\mathrm{pw}}$, respectively). For $\beta_{\mathrm{rc}}$, we compared the $\beta_{\mathrm{rc}}$ of each habitat to the $\beta_{\mathrm{rc}}$ of 1000 randomized communities with the same species richness $(* * * P<0.001$, Mann-Whitney test). For functional and phylogenetic beta diversity, we compared respective observed $D_{\mathrm{pw}}$ to the $D_{\mathrm{pw}}$ of null expectations (***P<0.001, NS, not significant; Welch's two-sample $t$ test). The boxes enclose the 25-75th percentiles of the values, the whiskers extend to 1.5 times the interquartile range.

ant communities in rubber plantations showed no phylogenetic signal with either index (Fig. 5).

There was also a sharp decline in phylogenetic diversity as a result of the decline in species richness (Fig. 1C), however, this decline was consistent with the null expectation for random assembled communities $(P=0.30$ and $P=0.32$ for forest and rubber plantations, respectively; Mann-Whitney test; Fig. 1E, also see NTI and NRI in Appendix S2: Fig. S2). The phylogenetic diversity of local forest and rubber habitats was also not significantly different from random expectations, which suggests that ants coexisting in the same habitat are phylogenetically random.

The pairwise phylogenetic beta matrix (phylogenetic $D_{\text {pw }}$ ) showed no differences between observed phylogenetic turnover and the phylogenetic turnover of random ant assemblies generated by the null model $(P=0.13$ and $P=0.35$ for forest and rubber plantations, respectively; Welch's two-sample $t$ test; Fig. 2C), and there was no clear separation in the NMDS plot (Fig. 2F). A similar phylogenetic beta diversity pattern was also found when using the nearest-neighbor phylogenetic beta matrix (phylogenetic $D_{\text {nn }}$; Appendix S2: Fig. S3). There also were no differences between observed and null expected phylogenetic gamma diversity in either habitat $(P=0.48$ and $P=0.67$ for forest and rubber plantations, respectively; Welch's two-sample $t$ test; Fig. 3D, E).

\section{Discussion}

Our results highlight changes to biodiversity within and across ecological communities associated with conversion from diverse tropical forest habitat to rubber monoculture, a rapidly emerging agroecosystem in Southeast Asia. We found a striking decline of ant species richness in rubber plantations as compared with nearby forest habitats. This pattern echoes previous findings showing habitat conversion from primary or secondary forests to monoculture plantations in many groups of organisms (Philpott et al. 2008a), including ants (Fayle et al. 2010).

This decline of alpha diversity was not random with respect to species identity; taxonomic beta diversity in rubber plantations was overall lower than would be expected if local communities were assembled by chance from the source pool. Moreover, taxonomic beta diversity was highest across forest $\times$ rubber community pairs than within either habitat, indicating a divergence in community structure. This was also confirmed by the NMDS analysis, which showed separation between ant communities sampled 
(A)

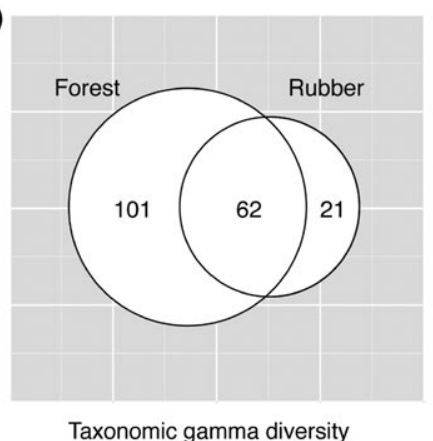

(B) 0

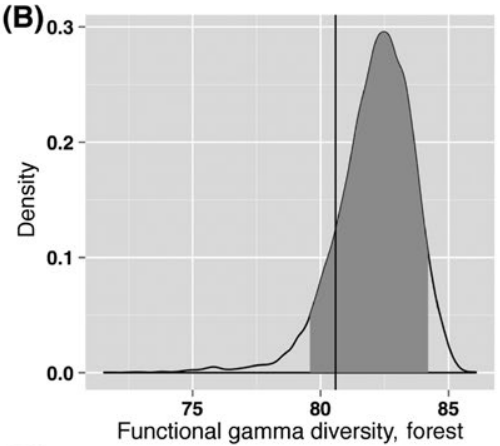

(D)

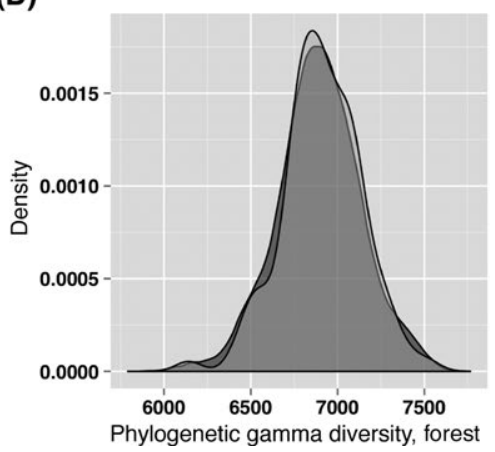

(C)

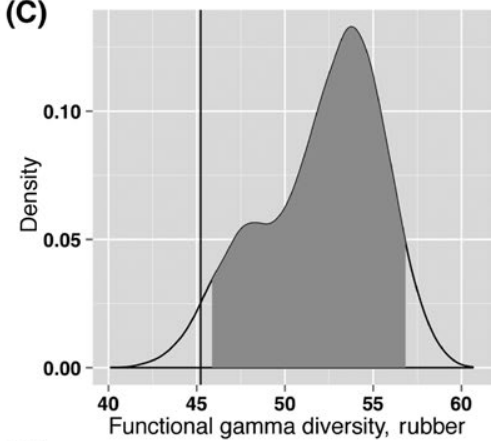

(E)

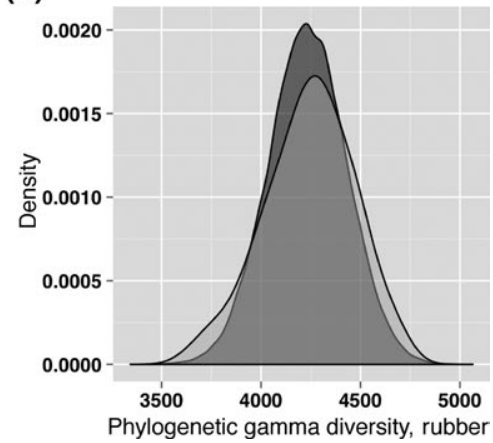

FIG. 3. Ant gamma diversities in different habitats. (A) Taxonomic gamma diversity in forest and rubber plantation. Venn diagram shows the number of species collected from each habitat, as well as the number of species shared in both habitats. For functional gamma diversity (B and C), vertical back lines are observed functional diversity, and distributions are the density of functional diversity derived from null model ( $n=10000$ randomizations) of the same species richness as observed communities. Shaded regions indicate $95 \%$ of the null distributions. For phylogenetic gamma diversity (D and E), distributions with light gray shading are the density of observed phylogenetic gamma diversity of 100 trees. The distributions with dark gray shading are the density of phylogenetic diversity derived from the null model $(n=1000$ randomizations for each of 100 trees) of the same species richness as observed communities. No significant differences were found between observed and null expected phylogenetic gamma diversity $(P=0.48, P=0.67$ for forest and rubber plantation, respectively; Welch's two-sample $t$ test $)$.

in forest and rubber habitats based on differences in species composition. These results support the hypothesis of environmental filtering, where the decrease of alpha diversity is due to the selective establishment of certain species that are tolerant of rubber habitat. Our results support the idea that anthropogenic disturbance, such as agriculture activities, and forest clearing increases the importance of deterministic, niche-based processes, leading to biotic homogenization (Vellend et al. 2007, Banks-Leite et al. 2012, Karp et al. 2012, Püttker et al. 2015).

In absolute terms, functional diversity was much reduced in rubber plantations compared with forest communities. This overall reduction pattern would be expected simply due to the decline of species richness (Flynn et al. 2009), but we found rubber habitats to be less functionally diverse than would be expected by chance indicating functional clustering in local communities. This pattern was also found on the landscape level; functional gamma diversity of ants in rubber plantation habitat, but not forest habitat, was lower than null expectations based on the entire pool of species. The lowerthan-expected functional beta diversity in rubber habitat showed that not only were local communities functionally simplified, but a similar functional structure was repeated in local communities across the landscape. Finally, there was an overall divergence in functional structure of ant communities among forest and rubber habitats that was not completely explained by species richness differences. This indicated the emergence of a functionally distinct community in rubber plantations. In total, these results support a more deterministic, niche-based species assembly process (environmental filtering) in rubber plantations with respect to functional traits.

Interestingly, our results showed that functional traits of ant communities are also clustered in forests with lower than expected functional alpha and beta diversities. While taxonomic beta diversity in forests is significantly higher than in rubber plantations, no differences were detected in functional beta diversity within the different forest and rubber plantation habitat types (after accounting for differences in species richness). These results indicate that analysis of taxonomic beta diversity alone may miss important aspects of community patterns. Indeed, it is possible to have two communities with very high species turnover but very low functional turnover (Swenson 2011a,b).

All the functional traits measured in Xishuangbanna ant communities exhibited phylogenetic signal, indicating 


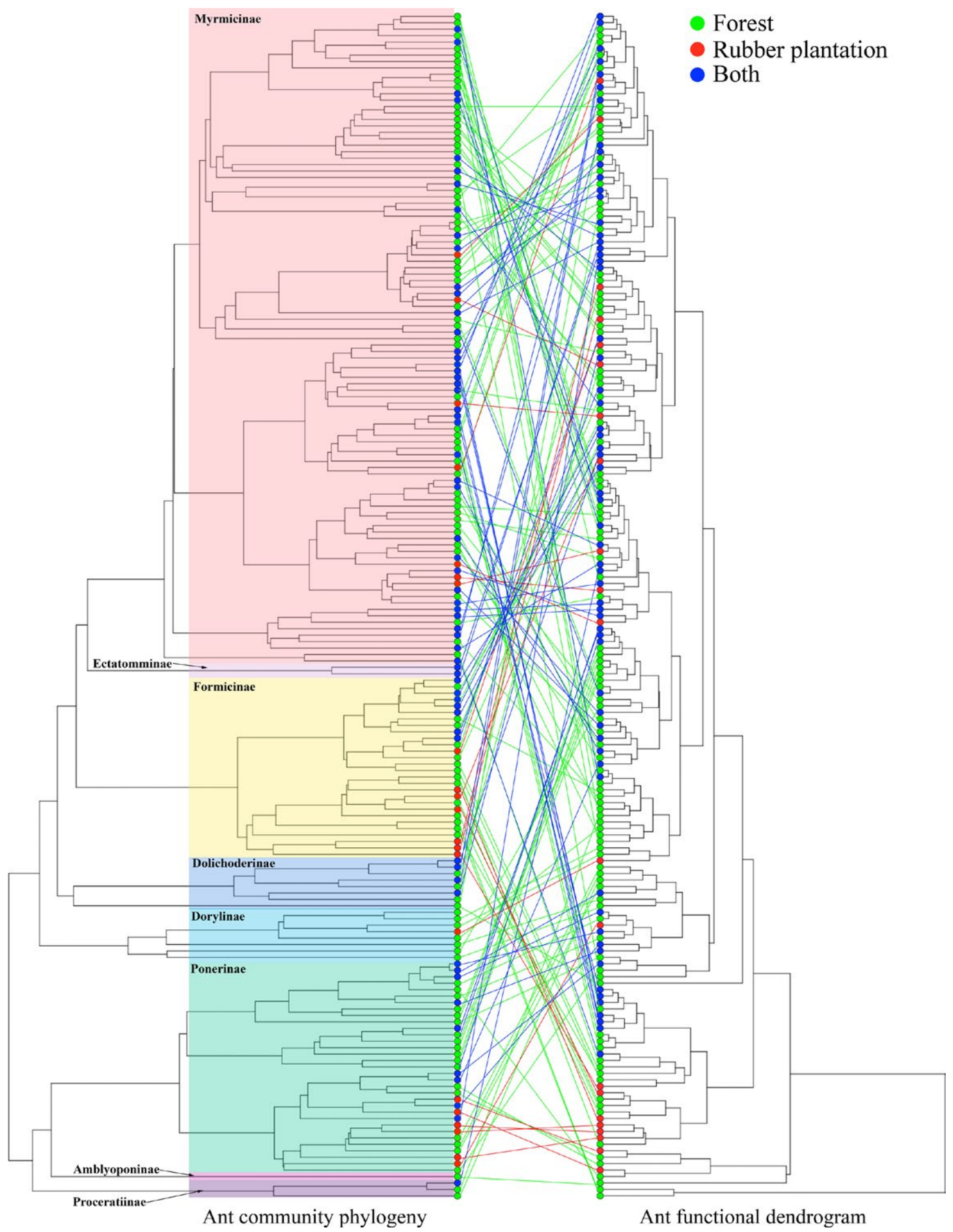

FIG. 4. The phylogeny (left) and functional dendrogram (right) of 184 ant species in this study. Color of circle indicates the habitat inhabited by that species. The lines link the same species between phylogeny and functional dendrogram.

that more closely related species share similar functional traits. These results are consistent with other studies, which found significant phylogenetic signal on ant body size (Machac et al. 2011, Donoso 2014). However, a recent study focused on Malagasy ants showed a lack of phylogenetic signal on most morphological traits (Blaimer et al. 2015). Overall, there is less phylogenetic signal in habitat preferences, however, Pagel's $\lambda$ detected 

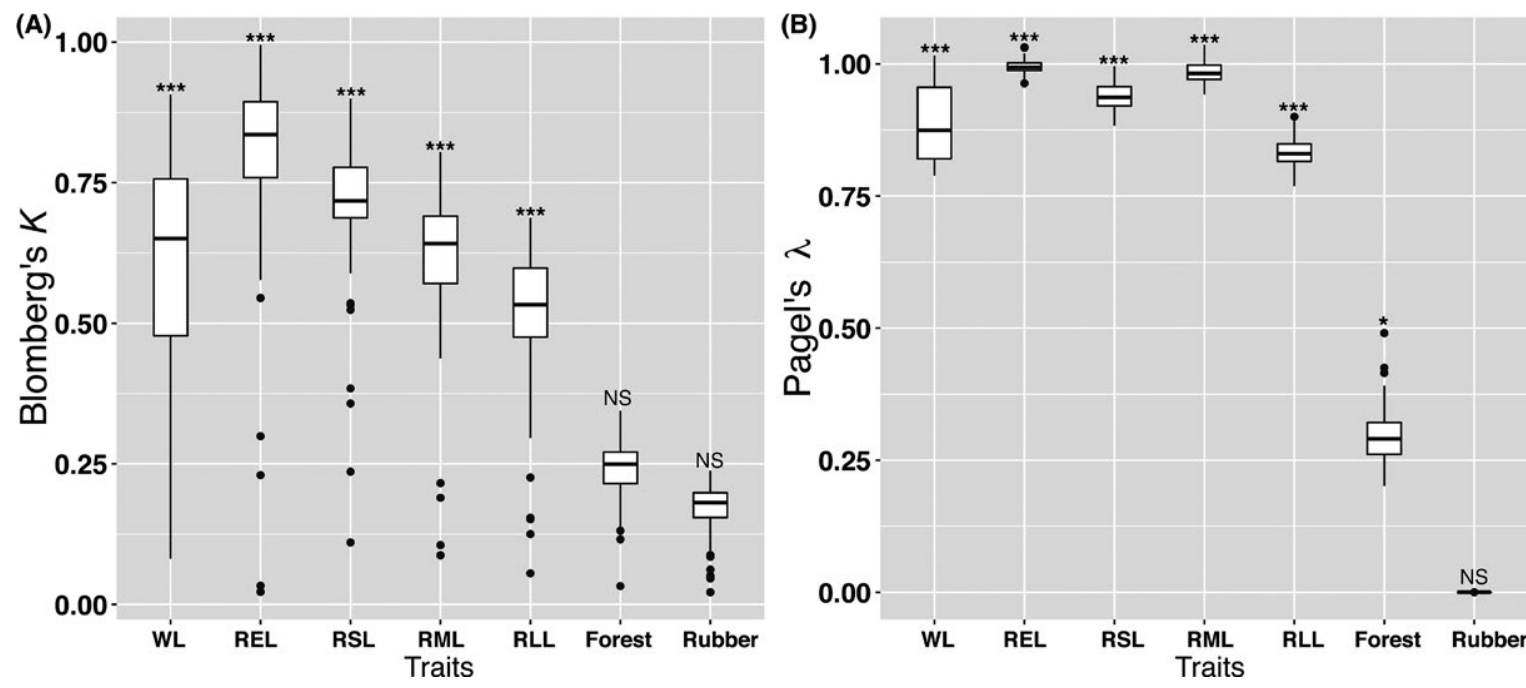

FIG. 5. Phylogenetic signal for functional traits and habitats. Phylogenetic signal was calculated using Blomberg's $K$ statistics (Blomberg et al. 2003), and Pagel's $\lambda$ (Pagel 1999). A value of $K / \lambda$ close to 1 indicates phylogenetic signal under Brownian motion, while a value of $K / \lambda$ close to 0 indicates no phylogenetic signal. The box encloses the $25-75$ th percentiles of the values, the whisker extends to 1.5 times the interquartile range. Abbreviations are WL, Weber's length; REL, relative eye length; RSL, relative scape length; RML, relative mandible length; RLL, relative leg length. Asterisks indicate statistical significance $(* * * P<0.001$, $* P<0.05)$.

slightly significant phylogenetic signal in forest habitats. This inconsistency between Blomberg's $K$ and Pagel's $\lambda$ may due to the fact that Pagel's $\lambda$ is a more sensitive measure of phylogenetic signal compared to Blomberg's $K$ (Münkemüller et al. 2012).

The results from measures of phylogenetic diversity were generally consistent with random community assembly with respect to phylogenetic structure. Ant communities in both forest and rubber plantation exhibited phylogenetic diversity consistent with null expectations. In addition, we did not find any differences between observed and expected phylogenetic beta diversity of ant communities from either natural forest or rubber habitats, indicating a random pattern of phylogenetic turnover that mirrors the null expectation.

Although a correlation was found between functional traits and phylogeny (e.g., significant phylogenetic signal), we did not find significant patterns of phylogenetic diversity in this study as we did in functional diversity. One possible explanation may be that the functional traits are not strongly conserved in our study (the $K$ values for all traits were below 1). Swenson (2011a) reported that the strong correlation between functional and phylogenetic beta diversity was only found when $K>2$. Kraft et al. (2007) also showed that phylogenetic local dispersion could mirror functional local dispersion when traits are very strongly conserved in the regional pool.

In summary, we found evidence for environmental filtering in taxonomic and functional dimensions of ant biodiversity, but random assembly in the phylogenetic dimension. The different patterns in functional and phylogenetic diversity in this study, even in the presence of phylogenetic signal, may indicate that using phylo- genetic dispersion as a proxy for trait dispersion and the underlying assembly processes could be misleading (Gerhold et al. 2015). Indeed, the aspects of phenotypes captured by functional and phylogenetic analyses are not equally relevant to community assembly. For example, the forest or rubber habitats may be amenable to certain combinations of functional traits as measured by our morphological variables, leading to functional clustering. At the same time, other phylogenetically correlated aspects of individual and colony-level phenotypes may lead to more intense competition between closely related species, leading to different patterns of phylogenetic diversity.

Interestingly, in contrast to previous findings from a rubber plantation in Cambodia (Hosoishi et al. 2013), we found a very limited presence of exotic and invasive ant species in rubber habitats at our site in China. Only a few exotic species were collected and with low occurrences, and most were also present in the forest habitats. One potential explanation for the discrepancy between our results and those of Hosoishi et al. (2013) lies in the differences in the scale of the rubber plantations in these studies; with the rubber plantation in Cambodia being a large, contiguous, industrial operation with limited nearby forest habitat, whereas, at our study sites, rubber plantations are interspersed with tracts and fragments of natural forest in a mixed landscape. Indeed, while richness is reduced in rubber habitats relative to native forest at our sites, it is possible that the presence of nearby native forest maintains a higher diversity of species in the rubber plantations than would otherwise not be able to persist in larger rubber monoculture. The presence of a more diverse native community could also explain 
the reduced impact of exotic species. These reasons for differences across the two studies remain speculative as replicated studies on the effects of landscape structure on agroecosystem communities are needed to rigorously address this question. This represents an interesting direction for future work.

From a conservation perspective, our results echo previous findings demonstrating that conversion from natural forest to rubber plantation is a pressing concern for biodiversity conservation in southeast Asia (Ziegler et al. 2009, Warren-Thomas et al. 2015). This is especially true due to the increasing demand of natural rubber worldwide driving the conversion from natural forest to rubber plantations (Warren-Thomas et al. 2015). It is likely that both a loss of species richness and the functional simplification of ant communities could have downstream effects on ecosystem processes and the populations of other insect groups. Studies have already suggested some solutions that could potentially minimize biodiversity and ecosystem function loss in rubber plantation, such as mixed-species agroecosystems, including native and crop species in order to increase the microhabitat heterogeneity (Phommexay et al. 2011). Such approaches have proven to be beneficial in other agroecosystems such as coffee plantations (Perfecto et al. 2005).

\section{Conclusions}

Understanding the effects of human agricultural activities on biodiversity is a pressing concern for ecology (Laurance et al. 2014). Our study found a suite of changes across different dimensions of biodiversity resulting from the conversion of natural forest to rubber plantations. Taken together, these results largely support a hypothesis of deterministic environmental filtering structuring communities in the agroecosystem. This likely reflects the strong ecological gradient formed by adjacent forest and plantation habitats. Variation in the sensitivity of ant species to habitat openness and disturbance is well documented (Economo and Sarnat 2012). This does not preclude the possibility that more stochastic, neutral dynamics dominate within similar habitats such as forests. To address such questions, it would be powerful to combine observational studies like this one with experimental manipulations, which are now increasingly common in plant community ecology (HilleRisLambers et al. 2012). Recently, researchers have started to test ant community assembly rules experimentally (Fowler et al. 2014, Fayle et al. 2015). Further research combining various approaches will benefit our understanding of the community ecology of ants in both natural habitats and agroecosystems, with consequences for basic and applied ecology.

Whether changes were consistent with null expectations, as with phylogenetic structure, or reflected deterministic reorganization, as with taxonomic and functional structure, we should not lose sight of the fact that biodiversity was much reduced in rubber plantation across all these dimensions. The links between diversity, community structure, and ecosystem functioning have long been suspected (Hooper et al. 2005) and now being demonstrated empirically through field studies in tropical environments (Ewers et al. 2015). Understanding the nature and effects of this biodiversity loss in ecologically dominant insect groups like ants remains a critical need for understanding the full consequences of the rapid emergence of agroecosystems such as rubber plantation.

\section{ACKNOWLEDGMENTS}

We would like to thank D. R. Yang and J. J. Liu for their assistance and advice on conducting this ant diversity survey. M. Ogasawara helped with specimen processing and B. Bolton, P. S. Ward, G. Fischer, S. Yamane, F. Hita Garcia, E. M. Sarnat, and M. Yoshimura helped with ant identifications. Thanks to N. Narula for the help with the phylogenies. We thank I. Perfecto and two anonymous reviewers for comments on the manuscript. The authors acknowledge the financial support of OIST and an NSF grant (NSF DEB-1145989).

\section{REFERENCES}

Andersen, A. N., and J. D. Majer. 2004. Ants show the way down under: invertebrates as bioindicators in land management. Frontiers in Ecology and the Environment 2:291-298.

Banks-Leite, C., R. M. Ewers, and J. P. Metzger. 2012. Unraveling the drivers of community dissimilarity and species extinction in fragmented landscapes. Ecology 93:2560-2569.

Bihn, J. H., G. Gebauer, and R. Brandl. 2010. Loss of functional diversity of ant assemblages in secondary tropical forests. Ecology 91:782-792.

Blaimer, B. B., S. G. Brady, T. R. Schultz, and B. L. Fisher. 2015. Functional and phylogenetic approaches reveal the evolution of diversity in a hyper diverse biota. Ecography 38:1-12.

Blomberg, S. P., T. Garland, A. R. Ives, and B. Crespi. 2003. Testing for phylogenetic signal in comparative data: behavioral traits are more labile. Evolution 57:717-745.

Brady, S., B. Fisher, T. Schultz, and P. Ward. 2014. The rise of army ants and their relatives: diversification of specialized predatory doryline ants. BMC Evolutionary Biology 14:93.

Cavender-Bares, J., K. H. Kozak, P. V. A. Fine, and S. W. Kembel. 2009. The merging of community ecology and phylogenetic biology. Ecology Letters 12:693-715.

Chase, J. M., and M. A. Leibold. 2003. Ecological niches: linking classical and contemporary approaches. University of Chicago Press, Chicago, Illinois, USA.

Chase, J. M., N. J. B. Kraft, K. G. Smith, M. Vellend and B. D. Inouye. 2011. Using null models to disentangle variation in community dissimilarity from variation in $\alpha$-diversity. Ecosphere 2:art24.

Del Toro, I., R. R. Ribbons, and S. L. Pelini. 2012. The little things that run the world revisited: a review of ant-mediated ecosystem services and disservices (Hymenoptera: Formicidae). Myrmecological News 17:133-146.

Donoso, D. A. 2014. Assembly mechanisms shaping tropical litter ant communities. Ecography 37:490-499.

Economo, E. P., and E. M. Sarnat. 2012. Revisiting the ants of Melanesia and the taxon cycle: historical and humanmediated invasions of a tropical archipelago. American Naturalist 180:E1-E16.

Ewers, R. M., et al. 2015. Logging cuts the functional importance of invertebrates in tropical rainforest. Nature Communication 6:6836. 
Faith, D. P.. 1992. Conservation evaluation and phylogenetic diversity. Biological Conservation 61:1-10.

Fayle, T. M., E. C. Turner, J. L. Snaddon, V. K. Chey, A. Y. C. Chung, P. Eggleton and W. A. Foster. 2010. Oil palm expansion into rain forest greatly reduces ant biodiversity in canopy, epiphytes and leaf-litter. Basic and Applied Ecology 11:337-345.

Fayle, T. M., P. Eggleton, A. Manica, K. M. Yusah, and W. A. Foster. 2015. Experimentally testing and assessing the predictive power of species assembly rules for tropical canopy ants. Ecology Letters 18:254-262.

Flynn, D. F. B., M. Gogol-Prokurat, T. Nogeire, N. Molinari, B. T. Richers, B. B. Lin, N. Simpson, M. M. Mayfield, and F. DeClerck. 2009. Loss of functional diversity under land use intensification across multiple taxa. Ecology Letters 12:22-33.

Fowler, D., J. P. Lessard, and N. J. Sanders. 2014. Niche filtering rather than partitioning shapes the structure of temperate forest ant communities. Journal of Animal Ecology 83:943-952.

Gerhold, P., J. F. Cahill, M. Winter, I. V. Bartish, and A. Prinzing. 2015. Phylogenetic patterns are not proxies of community assembly mechanisms (they are far better). Functional Ecology 29:600-614.

Gibb, H., J. Stoklosa, D. I. Warton, A. M. Brown, N. R. Andrew, and S. A. Cunningham. 2015. Does morphology predict trophic position and habitat use of ant species and assemblages?. Oecologia 177:519-531.

Gotelli, N. J., A. M. Ellison, R. R. Dunn, and N. J. Sanders. 2011. Counting ants (Hymenoptera: Formicidae): biodiversity sampling and statistical analysis for myrmecologists. Myrmecological News 15:13-19.

Guénard, B., and R. R. Dunn. 2012. A checklist of ants of China. Zootaxa 3558:1-77.

Guénard, B., and A. Lucky. 2011. Shuffling leaf litter samples produces more accurate and precise snapshots of terrestrial arthropod community composition. Environmental Entomology 40:1523-1529.

Hansen, M. C., et al. 2013. High-resolution global maps of 21stcentury forest cover change. Science 342:850-853.

HilleRisLambers, J., P. B. Adler, W. S. Harpole, J. M. Levine, and M. M. Mayfield. 2012. Rethinking community assembly through the lens of coexistence theory. Annual Review of Ecology, Evolution, and Systematics 43:227-248.

Hölldobler, B., and E. O. Wilson. 1990. The ants. Belknap, Cambridge, Massachusetts, USA.

Hooper, D. U., et al. 2005. Effects of biodiversity on ecosystem functioning: a consensus of current knowledge. Ecological Monographs 75:3-35.

Hosoishi, S., A. Le Ngoc, S. Yamane, and K. Ogata. 2013. Ant diversity in rubber plantation (Hevea brasiliensis) of Cambodia. Asian Myrmecology 5:69-77.

Hubbell, S. P. 2001. The unified neutral theory of biodiversity and biogeography. Princeton University Press, Princeton, New Jersey, USA.

Karp, D. S., A. J. Rominger, J. Zook, J. Ranganathan, P. R. Ehrlich, and G. C. Daily. 2012. Intensive agriculture erodes $\beta$-diversity at large scales. Ecology Letters 15:963-970.

Keller, R. A. 2011. A phylogenetic analysis of ant morphology (Hymenoptera: Formicidae) with special reference to the poneromorph subfamilies. Bulletin of the American Museum of Natural History 335:1-90.

Kembel, S. W., P. D. Cowan, M. R. Helmus, W. K. Cornwell, H. Morlon, D. D. Ackerly, S. P. Blomberg, and C. O. Webb. 2010. Picante: R tools for integrating phylogenies and ecology. Bioinformatics 26:1463-1464.

Kraft, N. J. B., W. K. Cornwell, C. O. Webb, and D. D. Ackerly. 2007. Trait evolution, community assembly, and the phylogenetic structure of ecological communities. American Naturalist 170:271-283.
Kuhn, T. S., A. Ø. Mooers, and G. H. Thomas. 2011. A simple polytomy resolver for dated phylogenies. Methods in Ecology and Evolution 2:427-436.

Kunstler, G., S. Lavergne, B. Courbaud, W. Thuiller, G. Vieilledent, N. E. Zimmermann, J. Kattge, and D. A. Coomes. 2012. Competitive interactions between forest trees are driven by species' trait hierarchy, not phylogenetic or functional similarity: implications for forest community assembly. Ecology Letters 15:831-840.

Laurance, W. F., J. Sayer, and G. C. Kenneth. 2014. Agricultural expansion and its impacts on tropical nature. Trends in Ecology and Evolution 29:107-116.

Levey, D. J., and M. M. Byrne. 1993. Complex ant-plant interactions: rain-forest ants as secondary dispersers and postdispersal seed predators. Ecology 74:1802-1812.

Liu, J. J., and J. W. F. Slik. 2014. Forest fragment spatial distribution matters for tropical tree conservation. Biological Conservation 171:99-106.

Liu, C., B. Guénard, F. Hita Garcia, S. Yamane, B. Blanchard, D. R. Yang, and E. Economo. 2015. New records of ant species from Yunnan, China. ZooKeys 477:17-78.

Lovette, I. J., and W. M. Hochachka. 2006. Simultaneous effects of phylogenetic niche conservatism and competition on avian community structure. Ecology 87:S14-S28.

Machac, A., M. Janda, R. R. Dunn, and N. J. Sanders. 2011. Elevational gradients in phylogenetic structure of ant communities reveal the interplay of biotic and abiotic constraints on diversity. Ecography 34:364-371.

Meng, L. Z., K. Martin, A. Weigel, and J. X. Liu. 2012. Impact of rubber plantation on carabid beetle communities and species distribution in a changing tropical landscape (southern Yunnan, China). Journal of Insect Conservation 16:423-432.

Moreau, C. S., and C. D. Bell. 2013. Testing the museum versus cradle tropical biological diversity hypothesis: phylogeny, diversification, and ancestral biogeographic range evolution of the ants. Evolution 67:2240-2257.

Moreau, C. S., C. D. Bell, R. Vila, S. B. Archibald, and N. E. Pierce. 2006. Phylogeny of the ants: diversification in the age of angiosperms. Science 312:101-104.

Mouchet, M., F. Guilhaumon, S. Villéger, N. W. H. Mason, J. A. Tomasini, and D. Mouillot. 2008. Towards a consensus for calculating dendrogram-based functional diversity indices. Oikos 117:794-800.

Münkemüller, T., S. Lavergne, B. Bzeznik, S. Dray, T. Jombart, K. Schiffers, and W. Thuiller. 2012. How to measure and test phylogenetic signal. Methods in Ecology and Evolution 3:743-756.

Myers, N., R. A. Mittermeier, C. G. Mittermeier, G. A. B. da Fonseca and J. Kent. 2000. Biodiversity hotspots for conservation priorities. Nature 403:853-858.

Oksanen, J., F. G. Blanchet, R. Kindt, P. Legendre, P. R. Minchin, R. B. O'Hara, G. L. Simpson, P. Solymos, M. H. H. Stevens and H. Wagner. 2015. Vegan: community ecology package. http://CRAN.R-project.org/package=vegan .

Pagel, M. 1999. Inferring the historical patterns of biological evolution. Nature 401:877-884.

Perfecto, I., J. Vandermeer, A. Mas, and L. S. Pinto. 2005. Biodiversity, yield, and shade coffee certification. Ecological Economics 54:435-446.

Petchey, O. L., and K. J. Gaston. 2006. Functional diversity: back to basics and looking forward. Ecology Letters 9:741-758.

Philpott, S. M., et al. 2008a. Biodiversity loss in Latin American coffee landscapes: review of the evidence on ants, birds, and trees. Conservation Biology 22:1093-1105.

Philpott, S. M., I. Perfecto, and J. Vandermeer. 2008b. Behavioral diversity of predatory arboreal ants in coffee agroecosystems. Environmental Entomology 37:181-191. 
Phommexay, P., C. Satasook, P. Bates, M. Pearch, and S. Bumrungsri. 2011. The impact of rubber plantations on the diversity and activity of understorey insectivorous bats in Southern Thailand. Biodiversity and Conservation 20:1441-1456.

Püttker, T., A. de Arruda Bueno, P. I. Prado, and R. Pardini. 2015. Ecological filtering or random extinction? Beta-diversity patterns and the importance of niche-based and neutral processes following habitat loss. Oikos 124:206-215.

R Development Core Team. 2015. R: a language and environment for statistics computing. R Foundation for Statistical Computing, Vienna, Austria. www.r-project.org

Revell, L. J. 2012. phytools: an R package for phylogenetic comparative biology (and other things). Methods in Ecology and Evolution 3:217-223.

Srivastava, D. S., M. W. Cadotte, A. A. M. MacDonald, R. G. Marushia, and N. Mirotchnick. 2012. Phylogenetic diversity and the functioning of ecosystems. Ecology Letters 15:637-648.

Swenson, N. G. 2011a. Phylogenetic beta diversity metrics, trait evolution and inferring the functional beta diversity of communities. PLoS ONE 6:e21264.

Swenson, N. G. 2011b. The role of evolutionary processes in producing biodiversity patterns, and the interrelationships between taxonomic, functional and phylogenetic biodiversity. American Journal of Botany 98:472-480.

Swenson, N. G. 2014. Functional and phylogenetic ecology in R. Springer, New York, New York, USA.

Swenson, N. G., et al. 2012. Phylogenetic and functional alpha and beta diversity in temperate and tropical tree communities. Ecology 93:S112-S125.
Tilman, D. 2001. Functional diversity. Pages 109-120 in S. A. Levin, editor. Encyclopedia of biodiversity. Volume 3. Academic Press, San Diego, California, USA.

Vellend, M., et al. 2007. Homogenization of forest plant communities and weakening of species-environment relationships via agricultural land use. Journal of Ecology 95:565-573.

Ward, P. S., S. G. Brady, B. L. Fisher, and T. R. Schultz. 2015. The evolution of myrmicine ants: phylogeny and biogeography of a hyperdiverse ant clade (Hymenoptera: Formicidae). Systematic Entomology 40:61-81.

Warren-Thomas, E., P. M. Dolman, and D. P. Edwards. 2015. Increasing demand for natural rubber necessitates a robust sustainability initiative to mitigate impacts on tropical biodiversity. Conservation Letters 8:1-13.

Webb, C. O., D. D. Ackerly, M. A. McPeek, and M. J. Donoghue. 2002. Phylogenies and community ecology. Annual Review of Ecology and Systematics 33:475-505.

Weinstein, B. G., B. Tinoco, J. L. Parra, L. M. Brown, J. A. McGuire, F. G. Stiles, and C. H. Graham. 2014. Taxonomic, phylogenetic, and trait beta diversity in South American hummingbirds. American Naturalist 184:211-224.

Weiser, M. D., and M. Kaspari. 2006. Ecological morphospace of New World ants. Ecological Entomology 31: 131-142.

Yang, Y., K. Tian, J. Hao, S. Pei, and Y. Yang. 2004. Biodiversity and biodiversity conservation in Yunnan, China. Biodiversity and Conservation 13:813-826.

Ziegler, A. D., J. M. Fox, and J. Xu. 2009. The rubber juggernaut. Science 324:1024-1025.

\section{SUPPORTING INFORMATION}

Additional supporting information may be found in the online version of this article at http://onlinelibrary.wiley.com/ doi/10.1890/15-1464.1/suppinfo 